\title{
Genetics of Type 1A Diabetes
}

\author{
Maria J. Redondo, Pamela R. Fain, and George S. Eisenbarth \\ Barbara Davis Center for Childhood Diabetes, University of Colorado Health Sciences Center, \\ 4200 East 9 th Avenue, Denver, Colorado 80262
}

\begin{abstract}
Type $1 \mathrm{~A}$ diabetes is an autoimmune disease with genetic and environmental factors contributing to its etiology. Twin studies, family studies, and animal models have helped to elucidate the genetics of autoimmune diabetes. Most of the genetic susceptibility is accounted for by human leukocyte antigen (HLA) alleles. The most-common susceptibility haplotypes are DQA $1 * 0301-\mathrm{DQB} 1 * 0302$ and DQA 1*0501-DQB1*0201. Less-common haplotypes such as DQA1*0401-DQB1*0402 and DQA I*0101-DQBI*0501 are associated with high risk for diabetes; however, large study populations are needed to analyze their effect. The DQA1*0102-DQB1*0602 haplotype is associated with diabetes resistance. DR molecules, such as DRBI*1401, confer protection from diabetes. Monozygotic twins of patients with type $1 \mathrm{~A}$ diabetes have a diabetes risk higher than that for HLA-identical ordinary siblings, suggesting that non-HLA genes contribute to diabetes risk. Polymorphisms in the regulatory region of the insulin gene (designated IDDM2), polymorphisms in cytotoxic T lymphocyte antigen-4 (CTLA-4) gene (IDDM12), and other genes are likely to contribute to diabetes risk and susceptibility in some individuals. In selected families, major diabetogenes (e.g., IDDM17, autoimmune regulator gene (AIRE)) are likely to be of importance. Other factors - either noninherited genes (i.e., somatic mutations and T-cell receptor or inmunoglobulin rearrangements) or environment - may have a rolc in progression to diabetes. This is suggested by the finding that the risk for monozygotic twins of patients with type $1 \mathrm{~A}$ diabetes is not 100 percent. Studying the genetics of type $1 \mathrm{~A}$ diabetes will allow us to better define this disease, to improve our ability to identify individuals at risk, and to predict the risk of associated disorders.
\end{abstract}

\section{Introduction}

Type 1A diabetes, formerly known as juvenile-onset diabetes or insulin-dependent diabetes mellitus (IDDM), is defined as immune-mediated diabetes (American Diabetes Association, 1997). Serum anti-islet autoantibodies, a marker of immune diabetes, are found in more than 90 percent of European patients with childhood-onset diabetes. Some cases of autoantibody-negative diabetes are clinically similar to those with positive autoantibodies (e.g., appear in lean, young individuals who need insulin therapy to avoid ketosis). At least some of these cases are likely to have immune-mediated diabetes in which current techniques cannot detect serum autoantibodies. The remaining cases of autoantibody-negative diabetes in children correspond to diabetes that is distinguishable from type 
1A diabetes: type 1B diabetes, type 2 diabetes, maturity onset diabetes of the young (MODY), cystic fibrosis-related diabetes, or other defined forms of diabetes. Type $1 \Lambda$ diabetes has been described among adults. Probably as many adults as children develop type $1 \mathrm{~A}$ diabetes, although type 2 diabetes is far more prevalent in adults than type $1 \mathrm{~A}$.

Knowlcdge of the genetics of type 1A diabetes allows better disease definition and improved ability to identify individuals at risk of diabetes and its associated disorders.

\section{Twin Studies}

Twin sludies help define the role of genetic and environmental factors in the etiology of disease (Martin et al., 1997; Phillips, 1993; Smith, 1974). In a primarily genetic disorder, concordance (i.e., both twins affected) is higher in monozygotic (identical) than dizygotic (fraternal) twins. Differences between monozygotic twins must be due to environmental factors, to genetic factors not coded in the germ line (e.g., somatic mutations), or to genes that undergo random rearrangement (e.g., immunoglobulin, T-cell receptor genes). Limitations of twin studies are small sample sizes, assignment of zygosity, and a patient-referral bias leading to over-ascertainment of concordant twin pairs (Hawkes, 1997; Phillips, 1993; Rowe and Leslie, 1995).

The problem of over-ascertainment has been addressed by analyzing twins identified through national registries (Petersen et al., 1997; Kyvik et al., 1995) and prospective studies of twins discordant for diabetes when recruited (Srikanta et al., 1983; Verge et al., 1995; Hawa et al., 1997). Concordance rates for type 1A diabetes have been reported among dizygotic twins of between zero percent (Hawa et al., 1997) and 13 percent (Kumar et al., 1993; Lorenzen et al., 1994; Redondo et al., 1999a). The concordance rate among monozygotic twins has been reported between 21 percent and 70 percent (Verge et al., 1995; Hawa et al., 1997; Srikanta et al., 1983; Kyvik et al., 1995; Redondo et al., 1999b). We have observed that monozygotic twins may become concordant many years after diagnosis in the index twin (the first twin to be diagnosed). Studies with long-term follow-up and lifetable projections have the highest concordance in monozygotic twins (Petersen et al., 1997; Verge et al., 1995). Nondiabetic monozygotic twins of patients with type $1 \mathrm{~A}$ diabetes show a high prevalence of anti-islet autoantibodies, between 42 and 76 percent (Verge et al., 1995; Hawa et al., 1997; Petersen et al., 1997). The appearance of multiple autoantibodies is followed in most instances by progression to diabetes (Verge et al., 1995; Hawa et al., 1997).

We recently assembled a series of nondiabetic dizygotic twins of patients with type 1A diabetes to compare with our series of monozygotic twins. Anti-islet autoantibody expression was analyzed in nondiabetic monozygotic twins $(n=53)$, dizygotic twins $(n=30)$, and siblings $(n=149)$ of patients with type 1 A diabetes, 
plus 101 controls (Redondo et al., 1999a). To reduce a potential patient-referral bias, we included only those pairs that were discordant at referral (i.e., one twin was diabetic and the other was not). Monozygotic twins had a much-higher risk of progressing to diabetes and expressing autoantibodies than did dizygotic twins. This finding is consistent with previous studies (Kaprio et al., 1992; Kumar et al., 1993; Hawa et al., 1997; Petersen et al., 1997) and supports the hypothesis of an important role for genetic factors in the determination of anti-islet autoimmunity. The risk for dizygotic twins and siblings was similar. This latter observation suggests that environmental factors, shared by dizygotic twins more than by ordinary siblings, does not increase progression to diabetes. There was also a higher penetrance of anti-islet autoimmunity in monozygotic twins who were heterozygous for the two high-risk human leukocyte antigen (HLA) haplotypes, DQ2/DQ8. Johnston et al. (1983) found a higher concordance for monozygotic twins with HLA DR3/DR4, the alleles most-frequently found on the same haplotype with DQ2/DQ8. This finding further supports the hypothesis that islet-cell autoimmunity may be predominantly genetically determined.

The observation that the concordance rate in monozygotic twins is not 100 percent indicates that nongermline (i.e., noninherited) genetic or environmental factors play a role. We recently combined two series, from Britain and the United States, to provide a database with 187 monozygotic twins of patients with type 1 A diabetes, with many twin pairs followed for up to 40 years (Redondo et al., 1999b). Progression to diabetes was similar in both series of monozygotic twins from the two countries. The hazard rate of progression to diabetes was higher within 10 years from diagnosis in the index twin, in line with observations by Olmos and coworkers (1998). However, progression to diabetes may happen many years after diagnosis in the index twin. In the combined series from Britain and the United States, 20 percent of the twins who progressed did so after more than 14 years of discordance, consistent with our previous studies (Verge et al., 1995). In addition we observed a higher progression rate to diabetes for twins whose index twin developed diabetes at a younger age.

Studies of identical twins of patients with type 1A diabetes provided the first evidence that the disorder developed chronically with progressive loss of firstphase insulin secretion (Srikanta, 1983). The observation that linear loss of firstphase insulin secretion preceded diabetes has been confirmed by Colman and coworkers (2000) in studies of first-degree relatives.

\section{Family Studies}

The observation of familial clustering of type $1 \mathrm{~A}$ diabetes is a strong indication that genetic factors are involved in the etiology of type 1A diabetes. Eightyfive percent of newly diagnosed cases are sporadic; that is, the patients do not have a first-degree relative with type $1 \mathrm{~A}$ diabetes. In spite of this, first-degree 
relatives of patients with type $1 \mathrm{~A}$ diabetes are at increased risk of developing positive autoantibodies and diabetes, compared to the general population. The familial clustering $\left(\lambda_{\mathrm{s}}\right)$ for a given disease can be calculated as the ratio of the risk for siblings divided by the prevalence in the general population (Risch et al., 1993; Todd and Farrall, 1996). The risk of type 1A diabetes in the general population is 0.4 percent, while the risk for siblings of affected individuals is approximately 6 percent. Thus, $\lambda_{\mathrm{s}}$ for type $1 \mathrm{~A}$ diabetes is approximately 15 times as great. The offspring of a mother with type 1A diabetes has a 1.3-4 percent risk of developing type $1 \mathrm{~A}$ diabetes. 'The risk for the offspring of a father with type $1 \mathrm{~A}$ diabetes is approximately six to nine percent (Warram et al., 1984; Tillil and Kobberling, 1987; Bleich et al., 1993). This parent-of-origin difference in transmission of diabetes is observed also for expression of anti-islet autoantibodies. The frequency and levels of expression of islet autoantibodies (glutamic acid decarboxylase (GAD)65, insulin antibodies (IAA), and islet cell antibodies (ICA)) were increased in 148 offspring of 103 fathers with diabetes, compared to 144 offspring of 102 mothers with diabetes (Yu et al., 1995).

The gender bias in transmission rate of diabetes has not been fully explained. Environmental hypotheses include maternal sex hormones modulating susceptibility in utero, immunologic tolerance to islet autoantigens, or selective fetal loss of potentially diabetic offspring (Warram et al., 1988). We did not find evidence for an effect of duration of diabetes in mothers before pregnancy nor the presence vs. the absence of diabetes at the time of the pregnancy (Yu et al., 1995). Genetic hypotheses for this parent-of-origin effect include genomic imprinting. Imprinting has been reported for a $19-\mathrm{kb}$ region in the insulin-like growth factor- 2 associated with diabetes risk (Baker et al., 1995). We found evidence for maternal imprinting of the insulin gene, with the B FOK allele of the insulin gene associated with protection only upon paternal transmission (Schwartz, 1990). It is not known how this observation correlates with the contradictory finding of a higher risk of diabetes transmission to offspring from diabetic fathers, compared to diabetic mothers.

\section{Genes Underlying Diabetes Susceptibility}

\section{A. MAJOR HISTOCOMPATIBILITY COMPLEX (MHC): DESCRIPTION AND NOMENCLATURE}

Peptides bound to MHC class I molecules are recognized by CD8 $\mathrm{T}$ cells. Antigens bound to MHC class II molecules are recognized by CD4 T cells. Nearly all nucleated cells express MHC class I molecules. MHC class II molecules are expressed by $\mathrm{B}$ lymphocytes, macrophages, and other antigen-presenting cells, and, in humans, by activated $\mathrm{T}$ lymphocytes. In addition, most epithelial cells can 
be induced to express class II molecules. The MHC molecules (in humans, also called HLA, human leukocyte antigen) are highly polymorphic; multiple different peptides can be presented to $T$ cells. MHC polymorphism results from different genes encoding families of MHC molecules, with each of these genes having multiple, germline-encoded alleles (Figure 1). Both MHC class I and class II moleculcs consist of two chains, alpha and beta. The alpha chain of MHC class I and both chains of MHC class II are encoded by genes within the MHC region on chromosome 6. The beta chain of MHC class I (beta-2-microglobulin), which is not polymorphic, is encoded by a genc on chromosome 15 . The MHC region in humans contains more than 100 genes, including HLA genes, extending over $4 \times 10^{6}$ base pairs of DNA. The alpha chains of three major MHC class I molecules are encoded by three different genes, HLA-A, HLA-B, and HLA-C. Each allele is identified by a unique number corresponding to a unique nucleotide sequence of the alpha chain (e.g., HLA-B0801).

The class II molecules, also known as immune response genes, are termed DR, DP, and DQ. Only the beta chain in DR molecules is polymorphic. Thus, different $D R$ molecules are identified by one number denoting the allele encoding the beta chain (e.g., DRB ${ }^{*} 0401$ ). Both beta and alpha chains in DQ molecules are polymorphic. The resulting DQ molecule is therefore identified by two numbers (e.g., DQA 1*0301, DQB1*0302). Additional genes within the MHC encode other molecules involved in the immune response, such as transporters associated

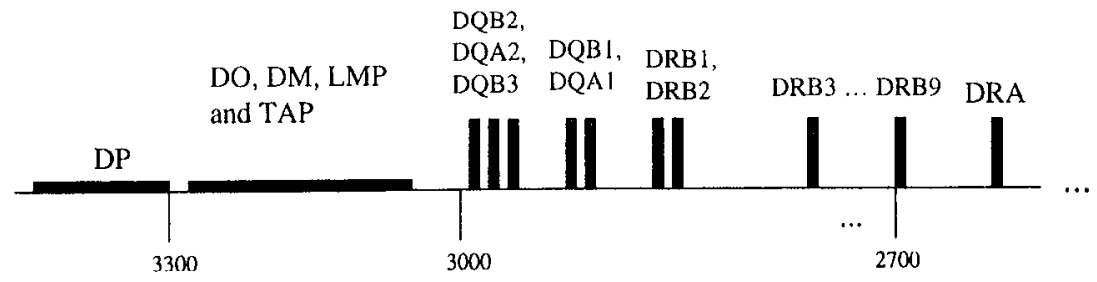

Class II

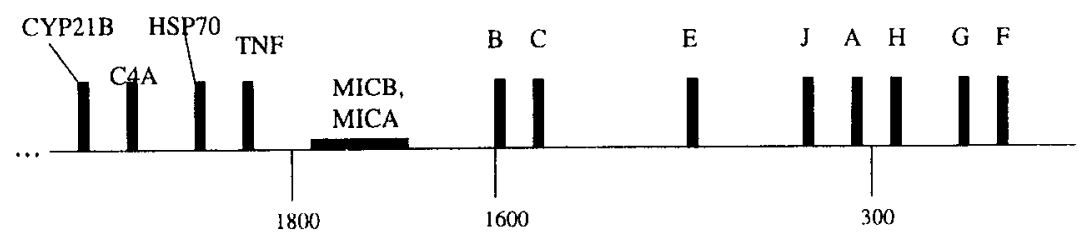

Class III

Class I

FIG. 1. Outline of the major histocompatibility complex region on chromosome 6 . 
with antigen processing (TAP-1 and TAP-2), the DM molecule, and proteosome subunits. The MHC class III region encodes complement and other genes.

\section{B. HLA AND TYPE 1A DIABETES}

\section{HLA Molecules Associated with Diabetes Susceptibility or Resistance}

The association of HLA alleles and type 1A diabetes was recognized more than 20 years ago by Nerup and co-workers (Nerup et al., 1974). Polyglandular failure syndrome type 2 , a constellation of autoimmune disorders associated with type $1 \mathrm{~A}$ diabetes, was also associated with HLA class I alleles, specifically, HLA-B8 (Eisenbarth et al., 1978). HLA class II alleles were found to be moresignificantly associated with type $1 \mathrm{~A}$ diabetes than class I alleles. It is currently believed that approximately half of the familial aggregation of type 1 diabetes is determined by HLA genes.

HLA DR 4 and DR3 are strongly associated with type 1A diabetes. However, this association is likely in part secondary to linkage disequilibrium (nonrandom association of alleles) between DR and DQ (Park et al., 1998). A haplotype is defined as a series of alleles of different genes on a chromosomal segment. HLA DRB1*0401, ${ }^{*} 0402$, and ${ }^{*} 0405$ alleles with DQA $1{ }^{*} 0301-\mathrm{DQB} 1{ }^{*} 0302$ (also called DQ8) are associated with high risk for diabetes. HLA DRB1*0301DQA 1*0501-DQB1*0201 (also called DQ2) is another high-risk haplotype. Ninety percent of individuals with type 1A diabetes have at least one of these two high-risk HLA haplotypes, compared to 20 percent of the general population. Approximately 35 percent of U.S. patients with type 1A diabetes are "DR3/DR4" heterozygotes, vs. only 2.4 percent of the general population. Overall, siblings of a patient with type $1 \mathrm{~A}$ diabetes have a risk of diabetes of approximately 6 percent. However, if a sibling is HLA identical to a sibling with type $1 \mathrm{~A}$ diabetes, the estimated risk increases to 20 percent. If the nonaffected sibling of a patient with diabetes carries both high-risk haplotypes DR4-DQA 1*0301-DQB1*0302 and HLA DR3-DQA $1 * 0501-\mathrm{DQB} 1 * 0201$, the risk of diabetes is approximately 25 to 40 percent. The risk conferred by the DQ8 molecule is modified by the DRB1 allele present on the same haplotype. The DRB $1 * 0402,{ }^{*} 0401$, and ${ }^{*} 0405$ alleles are associated with high susceptibility, the DRB ${ }^{*} 0404$ with moderate susceptibility, and the DRB1*0403 with "protection" against type 1A diabetes (Park et al., 1998). The presence of the HLA-DR4-DQ8 haplotype in first-degree relatives of patients with type 1A diabetes is associated with anti-insulin and ICA512 autoantibodies, both in individuals who developed diabetes on follow-up and in those who did not (Ziegler et al., 1991). In another study where 72 ICA-positive, first-degree relatives and 126 autoantibody-negative, first-degree relatives were studied, the frequency of the high-risk haplotypes was higher among ICA-positive relatives, compared to autoantibody-negative relatives (Pugliese et al., 1995). In 
addition, first-degree relatives with the high-risk allele DQB1*0302 progressed to diabetes at a younger age than relatives without this allele. The relatives with the high-risk allele DQB1*0201 without DQB 1*0302 progressed at an older age, compared to those relatives without it.

Uncommon HLA alleles also significantly affect diabetes risk of protection. However, large study populations are needed to identify their influence. The Human Biological Data Interchange (HBDI) type 1 repository has made it possible to analyze transmission of DQ haplotypes to affected and unaffected offspring from 276 families. The HBDI created a repository for DNA and cell lines in part for the purpose of mapping non-HLA genes associated with type 1A diabetes by linkage analysis. Over 95 percent of the HBDI families were multiplex; that is, ascertained with two or more affected siblings. Spurious association can result from artifacts of population structure, such as admixture or stratification. The transmission disequilibrium test (TDT) has been used to help distinguish between association due to linkage and spurious association (Spielman and Ewens, 1996). Under the null hypothesis of no linkage, a haplotype is expected to be transmitted 50 percent of the time from a heterozygous parent to an offspring. The TDT compares the observed transmission frequency to diabetic offspring against the expected 50 percent. A significant TDT result could be due to meiotic segregation distortion. If so, both affected and unaffected offspring would have this distortion. To rule out this possibility, the haplotype transmission frequencies to affected and unaffected offspring are compared by a $2 \times 2$ contingency chi-square test. We used the TDT analysis to assess the transmission of DQ haplotypes from heterozygous parents to children with diabetes and to their nondiabetic siblings. The rank order of transmission to affected offspring was DQA 1*0301-DQB1*0302 (associated with the highest risk), DQA1*0501-DQB 1*0201, DQA1*0401-DQB 1*0402, and DQA 1*0101-DQB $1 * 0501$. The DQ haplotype that was least-often transmitted to affected offspring was the well-known protective DQA 1*0102-DQB1*0602, followed by DQA 1*0103-DQB1*0603.

HLA alleles also confer protection from diabetes (Pugliese et al., 1995; Baisch et al., 1990). The best-known "protective" allele is DQB 1*0602, usually found on "DR2" (DRB1*1501-DQA1*0102-DQB1*0602) haplotypes. Approximately 20 percent of Americans and Europeans have DQA ${ }^{*} 0102-D Q B 1 * 0602$, while less than one percent of children with type $1 \mathrm{~A}$ diabetes carry these alleles. Table I illustrates the risk or protection associated with different HI.A alleles. The protection conferred by the DQA 1*0102-DQB1*0602 haplotype appears to be dominant, since it is protective even in the presence of a high-risk haplotype in the same individual. Of note, the frequency of the protective allele $\mathrm{DQB} 1 * 0602$ was similar among 72 ICA-positive, first-degree relatives and 126 first-degree relatives who were autoantibody negative (Pugliese et al., 1995). Progression to diabetes is, however, rare in 0602-positive, first-degree relatives, even in the 
TABLE 1

Risk of Type IA Diabetes Associated with DR and DQ Alleles

\begin{tabular}{|c|c|c|}
\hline DQAl & DQB1 & DRB \\
\hline \multicolumn{3}{|c|}{ High Risk } \\
\hline 0301 & 0302 & 0401 \\
\hline 0301 & 0302 & 0402 \\
\hline 0301 & 0302 & 0405 \\
\hline 0501 & 0201 & 0301 \\
\hline \multicolumn{3}{|c|}{ Moderate Risk } \\
\hline 0401 & 0402 & 0801 \\
\hline 0101 & 0501 & 0101 \\
\hline 0301 & 0303 & 0901 \\
\hline \multicolumn{3}{|c|}{ Weak or Moderate Protection } \\
\hline 0301 & 0302 & 0403 \\
\hline 0201 & 0201 & 0701 \\
\hline 0501 & 0301 & 1101 \\
\hline \multicolumn{3}{|c|}{ Strong Protection } \\
\hline 0102 & 0602 & 1501 \\
\hline 0101 & 0503 & 1401 \\
\hline 0201 & 0303 & 0701 \\
\hline
\end{tabular}

presence of positive autoantibodies. Interestingly, autoantibody-positive, 0602positive patients who did not develop diabetes had a restricted pattern of ICA staining, associated with high titers of GAD65 autoantibodies (Verge et al., 1996). Among ICA-positive, 0602-positive relatives, there is a more-limited response to islet antigens, often directed to GAD, compared to ICA-positive relatives without the 0602 allele (Gianani et al., 1992,1996).

A progressive decrease in first-phase insulin release (FPIR) on intravenous glucose tolerance testing (IVGTT) predicts progression to diabetes. DQB ${ }^{*} 0602$ positive, ICA-positive relatives were more likely to have a normal insulin secretion on IVGTT. In another study among individuals identified through the Dia- 
betes Prevention Trial, 0602-positive individuals were less likely to have positive insulin autoantibodies or low FPIR than relatives without DQB 1*0602 (Greenbaum et al., 2000). However, 29 percent of the ICA-positivc, 0602-positive relatives did have insulin autoantibodies or low FPIR, defined as below the 10th percentile. These findings suggest that the protective effect of the DQB $1 * 0602$ allele is likely to occur after the autoimmune destruction of the pancreas has begun. The presence of $\mathrm{DQB} 1 * 0602$ or sequence-related alleles protects from diabetes also in patients with stiff-man syndrome, who are otherwise at increased risk for type $1 \mathrm{~A}$ diabetes. Of note, this syndrome is associated with extremely high values of GAD autoantibodies.

It was reported that four patients with type $1 \mathrm{~A}$ diabetes who were initially found to have the conventional DQB1*0602 with standard sequence-specific oligonucleotide (SSO) typing techniques actually had novel variants of this allele with sequencing (Hoover and Marta, 1997). This finding would suggest that the conventional DQA $1{ }^{*} 0102-\mathrm{DQB} 1{ }^{*} 0602$ haplotype is never found in patients with type 1A diabetes and that this haplotype confers absolute protection from the disease. However, we have documented eight nondiabetic, ICA-positive, first-degree relatives and six patients with type 1 diabetes who carry the DQA ${ }^{*} 0102-$ DQB1*0602 haplotype. These individuals were first typed with standard SSO techniques, then confirmed by direct sequencing of the second exon of $\mathrm{DQB} 1^{*} 0602$ and DQA ${ }^{*} 0102$ alleles. All subjects carried conventional DQA 1*0102-DQB1*0602 exon 2 sequences (Pugliese et al., 1999). Type 1A diabetes can develop in individuals carrying the $\mathrm{DQB} 1{ }^{*} 0602$ allele, indicating that the protective effect associated with this allele is not absolute. The protective effect of DQB1*0602 may differ among ethnic groups. African American, ICApositive relatives were more likely to carry the $\mathrm{DQA} 1 * 0102-\mathrm{DQB} 1 * 0602$ haplotype than other ethnic groups. Hispanic, ICA-positive relatives with DQB $1 * 0602$ were more likely to have positive insulin autoantibodies or to have low FPIR than other racial groups (Greenbaum et al., 2000).

The DQB1*0602 allele is usually found on DR2-DRB1*1501-DQA 1*0102$\mathrm{DQB} 1{ }^{*} 0602$ haplotypes. However, protection from diabetes by this haplotype seems to be an effect of the DQB molecule. We described three siblings with type 1A diabetes who carried DR2 (Erlich et al., 1991). Utilizing sequence analysis, an unusual DQB1 allele was found on their DR2 haplotype, while the DRB1 alleles on these DR2 haplotypes was the conventional allele $\left({ }^{*} 1501\right)$. Further evidence was found in a recent study of 1371 subjects from the HBDI type 1 diabetes repository and 2441 subjects from the Norwegian Type 1 Diabetes Simplex Families (NODIAB) study (Redondo et al., 2000). DQA 1*0102$\mathrm{DQB} 1 * 0602$ haplotypes were infrequently transmitted to diabetic offspring $(2 / 313)$, while rare $\mathrm{DRB} 1 * 1501$ haplotypes without DQA $1 * 0102-\mathrm{DQB} 1 * 0602$ were transmitted to $5 / 11$ affected offspring. This finding again supports the hypothesis that the protection associated with the DQA1*0102-DQB1 ${ }^{*} 0602$ haplo- 
type is an effect of the DQ alleles rather than the DRB1*1501 in linkage disequilibrium.

$\mathrm{DR}$ alleles also may be protective against type $1 \mathrm{~A}$ diabetes. In the non-obesc diabetic (NOD) mouse, both I-E and I-A (DR and DQ molecules) protect from autoimmune diabetes (Yamane et al., 1996). Combining families from NODIAB and the HBDI, 728 patients with type 1A diabetes, and 110 healthy controls from the Barbara Davis Center provided us with a database to analyze the risk or protection associated with uncommon alleles. The HBDI study has been described above. The NODIAB study included 526 Norwegian families with one child diagnosed with diabetes before age 15. Less than three percent of families in this study had two affected children. We used the TDT analysis to analyze transmission to affected and unaffected offspring within fannilies in the HBDI and NODIAB studies. DQA1*0101-DQB ${ }^{*} 0503$ is a rare haplotype, present only in 1.6 percent of haplotypes in North America (Gjertson and Terasaki, 1998). This haplotype was transmitted from heterozygous parents to affected offspring only $2 / 42$ times. In the analysis of patients and controls, we also found that this allele is significantly less frequent among cases than controls. These findings are in agreement with previous reports (Ronningen et al., 1991). Interestingly, the only two affected offspring who inherited this haplotype in our combined family study did not have the DRB1*1401 allele usually found on the same haplotype with DQB I*0503, while all nonaffected children who inherited DQA ${ }^{*} 0101-$ DQB 1*0503 had DRB $1 * 1401$. Among the three patients with type 1A diabetes from the Barbara Davis Center who had DQB 1*0503, only one had DRB1*1401.

Polymorphisms in the heat shock protein genes located within the MHC are associated with type $1 \mathrm{~A}$ diabetes. However, this association is believed to be due to linkage disequilibrium of alleles on extended haplotypes associated with diabetes (Pugliese et al., 1992). Similarly, tumor necrosis factor (TNF) microsatellite haplotype associations with type I diabetes and multiple sclerosis were attributable to the known extended haplotype associations of these diseases (Garcia-Merino, et al., 1996).

\section{Mechanisms of Diabetogenicity of HLA Molecules}

The diabetes susceptibility or protection associated with HLA molecules may be related to their ability to present peptides of relevance to diabetogenic $T$ cells. Individuals with HLA molecules that are not able to effectively present specific peptides to naïve $T$ cells in the thymus might fail to engender tolerance. Alternatively, specific HLA alleles may selectively present an islet peptide to mature $T$ lymphocytes that have escaped negative selection. The two mechanisms - lack of central tolerance affecting the T-cell repertoire in the thymus or abnormal tolerance in the periphery - are not mutually exclusive and could coexist. The ability to present antigens may depend on conformational properties of the HLA 
molecule, with some alleles binding to peptides in such a fashion that the antigen would never be effectively presented to the $T$ cells. The DQA $1 * 0301$ allele frequently found on DR4 haplotypes, as well as *0101, *0102, *0103, and *0201 (lineage 1-3 DQA1 alleles), are associated with high serum levels of insulin autoantibodies (Pugliese et al., 1994b).

The hypothesis of conformational structure influencing diabetogenicity of some HLA alleles or haplotypes is further supported by inter-ethnic group studies. For instance, among the Japanese, the $\mathrm{DQB} 1{ }^{*} 0401$ allele is usually found on DR4-DQA 1*0301-DQB1*0401 haplotypes and is associated with diabetes risk (Aparicio et al., 1988). The DQB $1 * 0401$ and $\mathrm{DQB} 1 * 0402$ alleles differ only by one amino acid. Among Norwegians, the heterozygous DQA ${ }^{*} 0301$ DQB 1*0302/DQA 1*0401-DQB 1*0402 genotype appears to be associated with type 1 A diabetes. A very similar molecule could be formed among Japanese in cis (DR4-DQA 1*0301-DQB1*0401) and among Norwegians in trans (DQA 1*0301- DQB1*0402).

Animal models of type $1 \mathrm{~A}$ diabetes, such as the NOD mouse or transgenic mice, are helping in the identification of self antigens and MHC molecules associated with anti-islet autoimmunity. Sherwin and coworkers have demonstrated that transgenic mice expressing human DQ8 with beta-cell expression of the co-stimulatory molecule B7-1 develop type 1A diabetes (Wen et al., 2000). The crystal structure of the diabetogenic I- $\mathrm{A}^{\mathrm{g} 7}$ molecule, the only class II allele expressed by the NOD mouse, has been elucidated. The molecule has a "normal" class II binding cleft with a large pocket and the ability to bind multiple peptides (Corper et al., 2000). Santamaria and colleagues have defined the structure of a peptide mimotope that elicits the preferential expansion of beta-reactive $T$ cells in NOD mice (Anderson et al., 1999). Finally, a mouse with a transgene encoding a "diabetogenic" T-cell receptor is protected from diabetes by multiple "deleting" class II alleles (Schmidt et al., 1999).

The protective DQ haplotype, DQA 1*0102-DQB $1 * 0602$, has an aspartic acid at position 57 of the DQ beta chain. On the other hand, the two DQ haplotypes that determine the highest risk for diabetes, DQA 1*0301-DQB1*0302 and DQA $1{ }^{*} 0501-\mathrm{DQB} 1 * 0201$, do not have an aspartic acid residue at that position but rather alanine. It was hypothesized that the presence of aspartic acid at position 57 of the DQ-beta chain determines protection from diabetes (Morel et al., 1988). Further reports suggested that an arginine at position 52 of the DQ-alpha chain determined susceptibility to type $1 \mathrm{~A}$ diabetes, as it is found on the DQA1*0301DQB 1*0302 and DQA 1*0501-DQB1*0201 haplotypes (Gutierrez-Lopez, 1992; Khalil et al., 1990,1992). However, the DQA1*0401-DQB1*0402 haplotype is also associated with diabetes risk. Although it has arginine at position 52 of the DQ-alpha chain, it has aspartic acid at position 57 of the DQ-beta chain, as do DQB $1 * 0301$ and DQB $1 * 0303$ alleles associated with moderate risk. 


\section{Disorders Associated with Type 1A Diabetes}

Individuals with type $1 \mathrm{~A}$ diabetes experience other diseases at an increased frequency, compared with the general population. In most cases, that association is with autoimmune disorders. Most dramatic are the autoimmune polyendocrine syndromes (APS) type 1 and type 2. APS type 1 is a rare constellation of autoimmune disorders often diagnosed in infancy, with hypoparathyroidism, mucocutaneous candidiasis, Addison's disease, and hepatitis (Maes and Eisenbarth, 1999). This syndrome is associated with mutations of the autoimmune regulator gene (AIRE) on chromosome 21(Aaltonen et al., 1997; Nagamine et al., 1997) and has no association with HLA alleles. The basis for the association of the different disorders that constitute this syndrome is not yet clear. Therefore, APS-1 is an interesting case of HLA-independent autoimmune diabetes, although the diabetes is clinically similar to HLA-dependent type 1A diabetes and is associated with anti-islet autoantibodies such as GAD. However, the presence of GAD anti-islet autoantibodies in individuals with this syndrome does not seem to predict progression to diabetes, as it does in sporadic cases of type 1A diabetes (Husebye et al., 1997), while individuals with ICA512 (IA-2) autoantibodies frequently progress.

The disorders commonly associated in APS type 2 are Addison's disease, type 1A diabetes, autoimmune thyroid disease, gonadal failure, celiac disease, pernicious anemia, and vitiligo (Redondo and Eisenbarth, 1997). This syndrome is associated with HLA DR and DQ alleles. Table II illustrates the HLA association of disorders found in APS type 2.

Stiff-man syndrome (SMS) is a rare neurologic disorder characterized by rigidity of the body musculature, with spasms triggered by sensory or emotional stimuli with a characteristic electromyographic pattern. Autoantibodies against GAD are found in the serum and cerebroespinal fluid of 60 percent of patients with SMS. SMS is associated with DQB1*0201 (Pugliese et al., 1993).

Patients with type $1 \mathrm{~A}$ diabetes have an increased risk of celiac disease. This is a common autoimmune disorder associated with characteristic intestinal lesions that occur upon the ingestion of gliadin, a glutamine-rich protein present in wheat, rye, and barley. Typically, the lesions disappcar rapidly when this protein is removed from the diet. Patients may present with gastrointestinal complaints and poor growth. In rare instances, intestinal lymphoma may occur. Extra-intestinal lesions include skin and enamel lesions. Intestinal biopsy is the standard procedure for the diagnosis of this condition. The major autoantigen in celiac disease is transglutaminase, accounting for the presence of serum antiendomysial autoantibodies. We developed a radioassay for autoantibodies to tissue transglutaminase and found that transglutaminase autoantibodies are highly predictive of celiac disease. All patients (13/13) with an index greater than 0.7 had a positive intestinal biopsy (Bao et al., 1999b). Patients with transglutaminase autoantibodies at a titer 
TABLE II

Major HLA Associations of Disorders Found in Autoimmune Polyendocrine Syndrome Type 2

\begin{tabular}{lll} 
Disease & DRB Association & DQADQB Association \\
\hline Addison's disease & 0404 & $0301 / 0302$ \\
& 0301 & $0501 / 0201$ \\
& DR5 & $0501 / 0301$ \\
Graves' disease & 0301 & $0501 / 0201$ \\
Type 1A diabetes & $0401,0402,0404$ & $0301 / 0302$ \\
& 0301 & $0501 / 0201$ \\
Pernicious anemia & DR5 & \\
Celiac disease & 0301 & $0501 / 0201$ in "cis" \\
& DR5/DR7 & $0501 / 0201$ in "trans" \\
Myasthenia gravis & 0301 & $0501 / 0201$ \\
Sjogren syndrome & 0301 & $0501 / 0201$ \\
\hline
\end{tabular}

lower than 0.3 had a negative biopsy. Patients (49/49) with the conventional antiendomysial autoantibodies had positive transglutaminase antibodies. Susceptibility to celiac disease is mainly associated with the DQA 1*0501-DQB1*0201 haplotype. Interestingly, this heterodimer may be encoded either in cis, usually with a DR3 haplotype, or in trans, with DR7-DQA $1 * 0201-D Q B 1 * 0201$ on one chromosome and DR5-DQA1*0501-DQB1*0301 on the other (Bao et al., 1999a). The expression of transglutaminase antibodies was greatly increased (approximately one third) among patients with type $1 \mathrm{~A}$ diabetes homozygous for DQA $1 * 0501-D Q B 1 * 0201$.

Addison's disease is an uncommon condition associated with autoimmune failure of the adrenal gland that leads to fatigue, hyperpigmentation of the skin, abdominal pain, dehydration, low blood pressure, cardiovascular shock, and, if untreated, death. Adrenal cortex autoantibodies (ACA) are found in 43 to 84 percent (Soderbergh et al., 1996; Falorni et al., 1997) of patients with idiopathic adrenal failure. The major target of ACA is the steroidogenic enzyme P450c21 (21-hydroxylase). Autoantibodies against 21-hydroxylase have been documented in over 90 percent of patients with Addison's disease associated to APS-1 or APS-2 (Tanaka et al., 1997) and have not been found in nonautoimmune adrenal insufficiency and other conditions. We did not find positive 21-hydroxylase autoantibodies in any of 240 healthy controls. Approximately 1.5 percent of 
patients with type $1 \mathrm{~A}$ diabetes are positive for these autoantibodies. Five percent (10/208) of patients with type 1 diabetes heterozygous for DQ8 and DQ2 expressed 21-hydroxylase antibodies, compared to less than 0.5 percent in patients with type 1A diabetes who have neither DQ8 nor DQ2. Three of the 21-hydroxylase diabetic patients were found to have Addison's disease. The genotype DRB 1*0404-DQA 1*0301-DQB 1*0302/DRB1*0301-DQA 1*0501-DQB1*0201 was present in 14/21 patients with Addison's disease (8/12 with diabetes and 6/9 without diabetes or anti-islet antibodies), vs. 0.7 percent of the general population $(109 / 15,547)$ and 11 percent of patients with type $1 \mathrm{~A}$ diabetes without Addison's disease (62/578). Among patients with diabetes with DQA $1^{*} 0301-\mathrm{DQB} 1^{*} 0302$, Addison's disease was strongly associated with DRB $1 * 0404$. Eighty percent (12/15) of patients carrying DRB $1{ }^{*} 0404-\mathrm{DQA} 1{ }^{*} 0301-\mathrm{DQB} 1{ }^{*} 0302$ and positive for 21-hydroxylase autoantibody had Addison's disease, in contrast to 10 percent (1/10) 21-hydroxylase autoantibody-positive patients who had other DRB1 alleles (DRB1*0401 or DRB $1 * 0402)$ (Yu, 1999).

\section{Insulin Gene (IDDM2)}

Most of the genetic susceptibility for type 1A diabetes is determined by HLA genes. However, polymorphisms in a noncoding region of the insulin gene, on chromosome $11 \mathrm{pl}$, have long been known to be associated with diabetes risk (Bennett et al., 1995,1996; Bell et al., 1984). This locus has been termed IDDM2 and includes a variable nucleotide tandem repeat (VNTR) minisatellite located at the $5^{\prime}$ end of the insulin gene. There are three main VNTR classes defined by VNTR size: class I (26-63 repeats), class II (approximately 80 repeats), and class III (140-200 repeats). Each of these classes may be further divided. Homozygosity for class I VNTR determines high risk for diabetes, while class III VNTRs confer dominant protection. The VNTR class III allele is associated with higher expression of messenger RNA for insulin within the thymus (Pugliese et al,, 1997). One hypothesis is that expression of insulin within the thymus leads to negative selection (deletion) of autoreactive $T$ cells and thus to development of tolerance. VNTR class I alleles, associated with low levels of proinsulin and insulin in the thymus during fetal life and childhood, may fail to shape the T-cell repertoire and lead to the presence of anti-insulin autoreactive $T$ cells. A parent-of-origin effect has been described for IDDM2, with protective alleles protecting when paternally inherited (Pugliese et al., 1994a; Sospedra et al., 1998)

\section{IDDM3-17}

At least 15 more loci have been associated with type $1 \mathrm{~A}$ diabetes, in addition to HI.A (IDDM1) and the insulin VNTR (IDDM2) (Table III). For most associa- 
TABLE III

Defined and Putative IDDM loci

\begin{tabular}{|c|c|c|}
\hline Locus & Chromosome & Marker \\
\hline IDDMl & $6 \mathrm{p} 21.31$ & HLA \\
\hline IDDM2 & $11 \mathrm{p} 15.5$ & $5^{\prime}$ insulin VNTR \\
\hline IDDM3 & $15 q 26$ & D15S107 \\
\hline IDDM4 & $11 \mathrm{q} 13$ & Fibroblast growth factor-3 (FGF3) \\
\hline IDDM5 & $6 \mathrm{q} 24-27$ & D65476-D65448 \\
\hline IDDM6 & $18 \mathrm{q} 21$ & D18S64 \\
\hline IDDM7 & $2 q 31-33$ & $\mathrm{D} 2 \mathrm{~S} 152$ \\
\hline IDDM8 & $6 q 27$ & D6S 1590 \\
\hline IDDM9 & $3 q 21-q 25$ & D3S1303 \\
\hline IDDM 10 & $10 p 11-q 11$ & D10S193 \\
\hline IDDMII & $14 q 24.3-14 q 31$ & DD14567 \\
\hline IDDM12 & $2 q 33$ & CTLA-4 \\
\hline IDDM13 & $2 q 34$ & D2S164 \\
\hline IDDM15 & $6 \mathrm{q} 21$ & D65283 \\
\hline IDDM17 & $10 \mathrm{q} 25.1$ & D10S1681 \\
\hline No "IDDM" & $16 q$ & D1653098 \\
\hline No "IDDM" & $\operatorname{lq}$ & D15617 \\
\hline
\end{tabular}

tions, the responsible gene has not been identified. Of note, there are three more loci in addition to HLA located on chromosome 6, three loci on the short arm or chromosome 2 , and three loci on chromosome 10 . The polymorphic, cytotoxic, T-lymphocyte-associated antigen-4 (CTLA-4) gene on chromosome 2q33 (designated IDDM12) encodes a receptor expressed by activated T cells. CTLA-4 binds B7 molecules and limits the proliferative response of activated T cells. An A/G polymorphism in the first exon in CTLA4 results in an amino acid change (Thr/Ala). The presence of an alanine at codon 17 of CTLA4 has been associated with susceptibility to type $1 \mathrm{~A}$ diabetes, autoimmune thyroid disease, and primary biliary cirrhosis (Agarwal et al., 2000). The effect of IDDM12 is independent of other genetic markers of type 1A diabetes, such as HLA alleles or haplotypes, or 
the INS VNTR I/I risk genotype (van der Auwera et al., 1997). Ethnic heterogenity has been reported with strong effect in three Mediterranean-European populations (Italian, Spanish, and French), the Mexican American population, and the Korean population. There is a weak transmission deviation in the Caucasian American and no deviation in the British, Sardinian, and Chinese populations (Marron et al., 1997). Another study in the Danish population did not support IDDM12 as a type 1 diabetes susceptibility locus (Larsen et al., 1999).

In nonautoimmune BALB/c mice, CTLA-4 (CD152) is also a downregulator of T-cell activation responses. CD152 blockade unmasked islet cell-specific, autoreactive T cells. Conversely, CD152 blockade in NOD mice failed to regulate islet-specific, auto-reactive $T$-cell responses but enhanced the T-cell response to the exogenous, foreign antigen $\mathrm{KLH}$ in both nonautoimmune $\mathrm{BALB} / \mathrm{c}$ and autoimmune NOD mice. These results suggest that there is not a global defect in CD152-mediated regulation of peripheral T-cell immune responses in NOD autoimmune mice. Rather, there is a defect specific to $T$ cells recognizing self antigen (Piganelli et al., 2000). In the NOD mouse, macrophages, dendritic cells, and $T$ cells, but not $B$ cells, express lower basal levels of CD86. It has been proposed that this low level of CD86 expression contributes to a defective regulation of autoreactive $\mathrm{T}$ cells by preventing the full activation of $\mathrm{T}$ cells and therefore the upregulation of CTLA-4 (Dahlen et al., 2000). Protection against diabetes in the NOD mouse has been associated with increased CD40L and CTLA-4-expressing Th cells and with the generation of a CD40- $\mathrm{IgG}+\mathrm{B}$ cells. This study associated the induction by mycobacterial infection of regulatory CD45RBlo CD38 + Th cells with the ability to trigger deletion or anergy of peripheral, self-reactive lymphocytes and shutting down IgG+ B-cell responses (Martins and Aguas, 1999). Interestingly, in spontaneously diabetic BB rats, ex vivo, adenovirus-mediated CTLA4Ig gene transfer induced local immunomodulation, blocking autoimmune recurrence and rejection of pancreaticoduodenal grafts. The transferred CTLA4Ig gene was strongly expressed in both endocrine and exocrine tissues. These results indicate the potential utility of local CD28-B7 co-stimulatory blockade for prevention of alloimmune and autoimmune destruction of pancreatic grafts in type 1 diabetic hosts (Uchikoshi et al., 1999).

An alternative hypothesis to polygenic inheritance is that type $1 \mathrm{~A}$ diabetes is an oligogenic, heterogeneous disease. In this hypothesis, HLA is a major diabetogenic locus with relatively few non-HLA genes determining diabetes susceptibility (i.e., different genes causing disease in different families). We have studied a Bedouin Arab family with 21 members with type 1A diabetes. Based on a search for linkage, we identified a locus (termed IDDM17) contributing to type 1 diabetes mapping to the long arm of chromosome $10(10 \mathrm{q} 25$; nonparametric linkage $=$ $4.99, p=0.00004)$. In this family, HLA DR3 or DR4 plus IDDM17 is associated with a diabetes risk of 40 percent (Verge et al., 1998). 


\section{Summary}

Type 1A diabetes is an autoimmune disease with genetic and environmental factors contributing to its etiology. Most of the genetic susceptibility is accounted for by HLA alleles. The most-common susceptibility haplotypes are DQA ${ }^{*} 0301$ DQB 1*0302 and DQA 1*0501-DQB 1*0201. Other haplotypes (e.g., DQA $1 * 0401-\mathrm{DQB} 1 * 0402$ and DQA $1 * 0101-\mathrm{DQB} 1 * 0501$ ) are diabetogenic; strongly protective alleles (e.g., DQB1*0602) exist. Differential expression of insulin or proinsulin in the thymus may contribute to diabetes susceptibility. This autoimmune process also can result from mutations of a transcription factor (AIRE gene), independent of HLA alleles. It is likely that an abnormal presentation of antigens, potentially insulin or proinsulin, to $T$ cells either within the thymus or in the periphery, or both, results in an abnormal $\mathrm{T}$-cell repertoire or an abnormal immune response. Interestingly, protective HLA alleles continue to have an effect after the autoimmune process has started. The observation that the diabetes risk for monozygotic twins of patients with type 1A diabetes is higher than the diabetes risk for HLA-identical ordinary siblings supports the hypothesis of non-HLA genes contributing to diabetes risk. The non-HI A genetic susceptibility is probably explained by multiple genes, different in different families. In addition, the

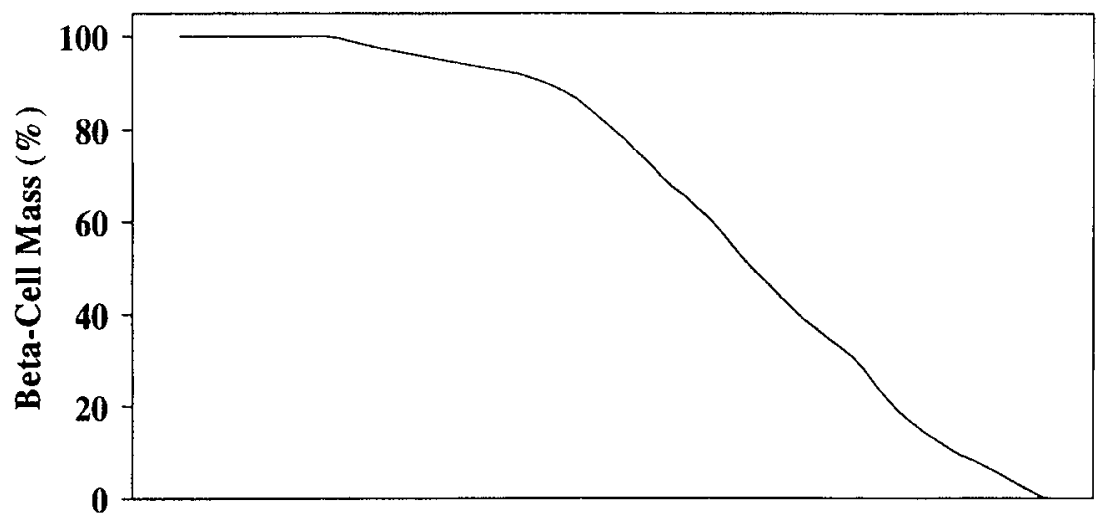

Time

Genetic Susceptibility

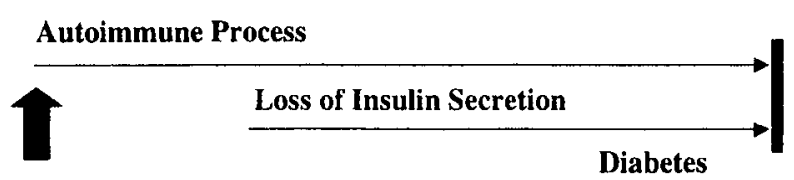

Triggering Factor(s)

FIG. 2. Hypothetical stages of the development of type 1A diabetes. 
observation that the risk for monozygotic twins of patients with type $1 \mathrm{~A}$ diabetes is not 100 percent indicates that there are other factors - either nongermline (noninherited) genes or environment - that have a role in progression to diabetes. Type $1 \mathrm{~A}$ diabetes is predictable, based on genetic, immunologic, and metabolic factors (Figure 2), such that trials of diabetes prevention are underway (e.g., Diabetes Prevention Trial 1 (DPT-1)). Several studies, including DAISY (Diabetes Autoimmunity Study of the Young) in the United States, suggest that diabetes will be predictable for individuals in the general population as well as first-degree relatives of patients. This should facilitate future preventive trials.

\section{REFERENCES}

Aaltonen, J., Bjorses, P., Perheentupa, J., Horelli-Kuitunen, N., Palotie, A., Peltonen, L., et al (1997). Nature Genet. 17, 399-403.

Agarwal, K., Jones, D.E., Daly, A.K., James, O.F., Vaidya, B., Pearce, S., et al. (2000). J. Hepatol. 32, 538-541.

American Diabetes Association. (1997). Diabetes Care 20, 1183-1197.

Anderson, B., Park, B.J., Verdaguer, J., Amrani, A., and Santamaria, P. (1999). Proc. Natl. Acad. Sci. U.S.A. 96, 9311-9316.

Aparicio, J.M.R., Wakisaka, A., Takada, A., Matsuura, N., and Aizawa, M. (1988). Immunogenetics 28, 240-246.

Baisch, J.M., Weeks, T., Giles, R., Hoover, M., Stastny, P., and Capra, J.D. (1990). N. Engl. J. Med. 322, 1836-1841.

Baker, B.S., Garioch, J.J., Bokth, S., Leonard, J., and Fry, L.J. (1995). J. Autoimmunity 8, 75-82.

Bao, F., Rewers, M., Scott, F., and Eisenbarth, G.S. (1999a). In "Endocrine and Organ Specific Autoimmunity" (G.S. Eisenbarth, ed.), pp. 85-96. R.G. Landes, Austin, Tex.

Bao, F., Yu, L., Babu, S., Wang, T., Hoffenberg, E.J., Rewers, M., et al. (1999b). J. Autoimmunity 13, 143-148.

Bell, G.I., Horita, S., and Karam, J.H. (1984). Diabetes 33, 176-183.

Bennett, S.T., Lucassen, A.M., Gough, S.C.L., Powell, E.E., Undlien, D.E., Pritchard, L.E., et al. (1995). Nature Genet. 9, 284-292.

Bennett, S.T., Wilson, A.J., Cucca, F., Nerup, J., Pociot, F., McKinney, P.A., et al. (1996). J. Autoimmunity 9, 415-421

Bleich, D., Polak, M., Eisenbarth, G.S., and Jackson, R.A. (1993). Diabetes 42, 1433-1439.

Colman, P.G., McNair, P., Steele, C., Gellert, S., Tait, B., Honeyman, M., et al. (2000). Diabetes 49, A36-A36 (abstract)

Corper, A.L., Stratmann, T., Apostolopoulos, V., Scott, C.A., Garcia, K.C., Kang, A.S., et al. (2000). Science 288, 505-511.

Dahlen, E., Hedlund, G., and Dawe, K. (2000). J. Immunol. 164, 2444-2456.

Eisenbarth, G.S., Wilson, P., Ward, F., and Lebovitz, H.E. (1978). N. Fngl I. Med. 298, 92-94.

Erlich, H.A., Griffith, R.L., Bugawan, T.L., Ziegler, R., Alper, C., and Eisenbarth, G.S. (1991). Diabetes 40, 478-481.

Falorni, A., Laureti, S., Nikoshkov, A., Picchio, M.L., Hallengren, B., Vandewalles, C.L., et al. (1997). Clin. Exp. Immunol. 107, 341-346.

Garcia-Merino, A., Alper, C.A., Usuku, K., Marcus-Bagley, D., Lincoln, R., Awdeh, Z., et al. (1996). Hum. Immunol. 50, 11-21.

Gianani, R., Pugliese, A., Bonner-Weir, S., Shiffrin, A.J., Soeldner, J.S., Erlich, H., et al. (1992). Diabetes 41, 347-353. 
Gianani, R., Verge, C.F., Moromisato-Gianani, R.I., Yu, L., Zhang, Y.J., Pugliese, A., et al. (1996). J. Autoimmunity 9, 423-425.

Gjertson, D.W., and Terasaki, P.I. (eds.) (1998). "HLA." American Society of Immunocompatibility and Genetics, Lenexa, Tex.

Greenbaum, C.J., Cuthbertson, D., Eisenbarth, G.S., Schatz, D.A., Zeidler, A., and Krischer, J.P. (2000). J. Clin. Endocrinol. Metab. 85, 1255-1260.

Gutierrez-Lopez, M.D., Bertera, S., Chantres, M.T., Vavassori, C., Dorman, J.S., Trucco, M., et al. (1992). Diabetologia 35, 583.588.

Hawa, M., Rowe, R., Lan, M.S., Notkins, A.L., Pozzilli, P., Christie, M.R., et al. (1997). Diabetes 46, $1270-1275$.

Hawkes, C.H. (1997). Diabetic Med. 17, 347-352.

Hoover, M.L., and Marta, R.T. (1997). Scand. J. Immunol. 45, 193-202.

Husebye, E.S., Gebre-Medhin, G., Tuomi, T., Perheentupa, J., Landin-Olsson, M., Gustafsson, J., et al . (1997), J. Clin. Endocrinol. Metab. 82, 147-150.

Johnston, C., Pyke, D.A., Cudworth, A.G., and Wolf, E. (1983). Br. Med. J. 286, 253-255.

Kaprio, J., Tuomilehto, J., Koskenvuo, M., Romanov, K., Reunanen, A., Eriksson, J., et al. (1992). Diabetologia 35, 1060-1067.

Khalil, I., D'Auriol, L., Gobet, M., Morin, L., Lepage, V., Deschamps, I., et al. (1990). J. Clin. Invest. 85, 1315-1319

Khalil, I., Deschamps, I., Lepage, V., Al-Daccak, R., Degos, L., and Hors, J. (1992). Diabetes 41, 378-384.

Kumar, D., Gemayel, N.S., Deapen, D., Kapadia, D., Yamashita, P.H., Lee, M., et al. (1993). Diabetes 42, 1351-1363

Kyvik, K.O., Green, A., and Beck-Nielsen, H. (1995), Br. Med. J. 311, 913-917.

Larsen, Z.M., Kristiansen, O.P., Mato, E., Johannesen, J., Puig-Domingo, M., de Leiva, A., et al. (1999). Autoimmunity 31, 35-42.

Lorenzen, T., Pociot, F., Hougaard, P., and Nerup, J. (1994). Diabetologia 37, 321-327.

Maes, M, and Eisenbarth, G.S. (1999). In "Contemporary Endocrinology on Autoimmune Endocrinopathies" (R. Volpé, ed.), pp. 349-363. Humana Press, Inc., Totowa, N.J.

Marron, M.P., Raffel, L.J., Garchon, H.J., Jacob, C.O., Serrano-Rios, M., Martinez, L.M., et al. (1997). Hum. Mol. Genet. 6, 1275-1282.

Martin, N., Boomsma, D., and Machin, G. (1997). Nature Genet. 17, 387-392.

Martins, T.C., and Aguas, A.P. (1999). Immunology 96, 600-605.

Morel, P.A., Dorman, J.S., Todd, J.A., McDevitt, H.O., and Trucco, M. (1988). Proc. Natl. Acad. Sci. U.S.A. 85, $8111-8115$.

Nagamine, K., Peterson, P., Scott, H.S., Kudoh, J., Minoshima, S., Heino, M., et al. (1997). Nature Genet. 17, 393-398.

Nerup, J., Platz, P., Anderson, O.O., Christy, M., Lyngsoe, J., Poulsen, J.E., et al. (1974). Lancet ii, 864-866.

Olmos, P., A'Hearn, R., Heaton, D.A., Millward, B.A., Risley, D., Pyke, D.A., et al. (1988). Diabetologia 31, 747-750.

Park, Y.S., Wang, C.Y., Ko, K.W., Yang, S.W., Park, M., Yang, M.C., et al. (1998). Hum. Immunol. 59, 794-801.

Petersen, J.S., Kyvik, K.O., Bingley, P.J., Gale, E.A.M., Green, A., Dyrberg, T., et al. (1997). Br. Med. J. 314, 1575-1579.

Phillips, D.I.W. (1993). Lancet 341, 1008-1009

Piganelli, J.D., Poulin, M., Martin, T., Allison, J.P., and Haskins, K. (2000). J. Autoimmunity 14, 123-131.

Pugliese, A., Awdeh, Z., Galluzzo, A., Yunis, E.J., Alper, C.A., and Eisenbarth, G.S. (1992). Diabetes 41, 788-791. 
Pugliese, A., Solimena, M., Awdeh, Z.L., Alper, C.A., Bugawan, T., Erlich, H.A., et al. (1993). J. Clin. Endocrinol. Metab. 77, 1550-1553.

Pugliese, A., Awdeh, Z.L., Alper, C.A., Jackson, R.A., and Eisenbarth, G.S. (1994a). J. Autoimmunity 7, 687-694.

Pugliese, A., Bugawan, T., Moromisato, R., Awdeh, Z.L., Alper, C.A., Jackson, R.A., et al. (1994b). J. Clin. Invest. 93, 2447-2452.

Pugliese, A., Gianani, R., Moromisato, R., Awdeh, Z.L., Alper, C.A., Erlich, H.A., et al. (1995). Diabetes 44, 608-613.

Pugliese, A., Zeller, M., Fernandez, A., Zalcberg, L.J., Bartlett, R.J., Ricordi, C., et al. (1997). Nature Genet. 15, 293-297.

Pugliese, A., Kawasaki, E., Zeller, M., Yu, L., Babu, S., Solimena, M., et al. (1999). J. Clin. Endocrinol. Metab. 84, 1722-1728

Redondo, M.J., and Eisenbarth, G.S. (1997). In "Endocrine and Organ Specific Autoimmunity" (G.S. Eisenbarth, ed.), pp. 41-61. R.G. Landes Company, Austin, Tex.

Redondo, M.J., Kawasaki, E., Mulgrew, C.L., Noble, J., Erlich, H., Freed, J., et al. (2000). J. Clin. Endocrinol. Metab. 85, 3793-3797.

Redondo, M.J., Rewers, M., Yu, L., Garg, S., Pilcher, C.C., Elliott, R.B., et al. (1999a). Br. Med. J. 318, 698-702.

Redondo, M.J., Yu, L., Hawa, M., Leslie, D.G., and Eisenbarth, G.S. (1999b). Diabetes 48, 780-780 (abstract).

Risch, N., Ghosh, S., and Todd, J.A. (1993). Am. J. Hum. Genet. 53, 702-714.

Ronningen, K.S., Spurkland, A., Iwe, T., Vartdal, F., and Thorsby, E. (1991). Tissue Antigens 37, 105-111.

Rowe, R.E., and Leslie, R.D.G. (1995). Diabetes Metab. Rev. 11, 121-136.

Schmidt, D., Amrani, A., Verdaguer, J., Bou, S., and Santamaria, P. (1999). J. Immunol, 162, 4627-4636.

Schwartz, R. (1990). N. Engl. J. Med. 323, 340-342.

Smith, C. (1974). Am. J. Hum. Genet. 26, 454-466.

Soderbergh, A., Winqvist, O., Norheim, I., Rorsman, F., Husebye, E.S., Dolva, O., et al. (1996). Clin. Endocrinol. (Oxf.) 45, 453-460.

Sospedra, M., Ferrer-Francesch, X., Dominguez, O., Juan, M., Foz-Sala, M., and Pujol-Borrell, R. (1998). J. Immunol. 161, 5918-5929.

Spielman, R.S., and Ewens, W.J. (1996). Am. J. Hum. Genet. 59, 983-989.

Srikanta, S., Ganda, O.P., Eisenbarth, G.S., and Soeldner, J.S. (1983). N. Engl. J. Med. 308, 322-325.

Tanaka, H., Perez, M.S., Powell, M., Sanders, J.F., Sawicka, J., Chen, S., et al. (1997). J. Clin. Endocrinol. Metab. 82, 1440-1446.

Tillil, H., and Kobberling, J. (1987). Diabetes 36, 93-99.

Todd, J.A., and Farrall, M. (1996). Hum. Mol. Genet. 5, 1443-1448.

Uchikoshi, F., Yang, Z.D., Rostami, S., Yokoi, Y., Capocci, P., Barker, C.F., et al. (1999). Diabetes 48, 652-657.

van der Auwera, B.J., Vandewalle, C.L., Schuit, F.C., Winnock, F., De Leeuw, I.H., Van Imschoot, S., et al. (1997). Clin. Exp. Immunol. 110, 98-103.

Verge, C.F., Gianani, R., Yu, L., Pietropaolo, M., Smith, T., Jackson, R.A., et al. (1995). Diabetes 44, 1176-1179.

Verge, C.F., Gianani, R., Kawasaki, E., Yu, L., Pietropaolo, M., Jackson, R.A., et al. (1996). Diabetes 45, 926-933.

Verge, C.F., Vardi, P., Babu, S., Bao, F., Erlich, H.A., Bugawan, T., et al. (1998). J. Clin. Invest. 102, 1569-1575

Warram, J.H., Krolewski, A.S., Gottlieb, M.S., and Kahn, C.R. (1984). N. Engl. J. Med. 311, 149-152.

Warram, J.H., Kroleski, A.S., and Kahn, C.R. (1988). Diabetes 37, 1328-1334.

Wen, L., Wong, F.S., Tang, J., Chen, N.Y., Altieri, M., David, C., et al. (2000). J. Exp. Med. 191, 97-104. 
Yamane, K., Yamamoto, K., Yoshikawa, Y., and Sasazuki, T. (1996). Clin. Exp. Immunol. 103, 141-148. Yu, L., Chase, H.P., Falorni, A., Rewers, M., Lernmark, $\AA$., and Eisenbarth, G.S. (1995). Diabetologia 38, 1353-1357.

Yu, L., Brewer, K.W., Gates, S., Wang, T., Babu, S., Gottlieb, P.A., et al. (1999). J. Clin. Endocrinol. Metab. 84, 328-335.

Ziegler, R., Alper, C.A., Awdeh, Z.L., Castano, L., Brink, S.J., Soeldner, J.S., et al. (1991). Diabetes 40, 709-714. 
